\title{
Multi-qudit states generated by unitary braid quantum gates based on Temperley-Lieb algebra
}

\author{
C.-L. Ho $\mathrm{H}^{1,2}$ and T. Deguchi ${ }^{1}$ \\ ${ }^{1}$ Department of Physics, Faculty of Core Research, Ochanomizu University \\ 2-1-1 Ohtsuka, Bunkyo-ku, Tokyo 112-8610, Japan \\ ${ }^{2}$ Department of Physics, Tamkang University, Tamsui 25137, Taiwan, R.O.C.*
}

\begin{abstract}
Using a braid group representation based on the Temperley-Lieb algebra, we construct braid quantum gates that could generate entangled $n$-partite $D$-level qudit states. $D$ different sets of $D^{n} \times D^{n}$ unitary representation of the braid group generators are presented. With these generators the desired braid quantum gates are obtained. We show that the generalized GHZ states, which are maximally entangled states, can be obtained directly from these braid quantum gates without resorting to further local unitary transformations. We also point out an interesting observation, namely for a general multi-qudit state there exists a unitary braid quantum gate based on the Temperley-Lieb algebra that connects it from one of its component basis states, if the coefficient of the component state is such that the square of its norm is no less than $1 / 4$.
\end{abstract}

PACS numbers: 03.67.Bg, 02.10.Kn, 03.67.Mn

Keywords: quantum entanglement, braid operators, Temperley-Lieb algebra

\section{INTRODUCTION}

Topological quantum computing is an approach to fault-tolerant quantum computing based on topological phases of matter. Most models of this approach are based on the braiding and fusing of anyons, quasi-particles which obey fractional statistics in two dimensions [1-4]. Interest in this approach is enhanced in recent years by the prospect that anyons could be practically realized through Majorana zero modes [5 7] in topological insulators [8, 9].

These interesting developments in quantum computation using the braiding of anyons have stimulated interest in applying the theory of braid groups to the fields of quantum information and quantum computation. In this respect, an interesting result is the realisation that a specific solution of the Yang-Baxter equation, the so-called Bell basis change matrix, can be obtained from the braiding relations [10]. It is known that this operator is a universal gate for quantum computing in the presence of local unitary transformations. As such, the result shown in 10 implies that in principle all quantum gates can be constructed from braiding operators together with single qubit gates.

The braid operator presented in [10] involves a solution of the Yang-Baxter equation. It generates the four maximally entangled Bell states from the standard basis of separable states. This has led to further investigation on the possibility of generating other entangled states by appropriate braiding operators [11 15]. For instance, in [12] unitary braiding operators based on the Yang-Baxter equation were used to realise entanglement swapping and generate the Greenberger-Horne-Zeilinger (GHZ) state [16] and the linear cluster state 17. The GHZ state is of fundamental importance as it is the maximally entangled multipartite state, which includes the Bell states as special cases.

In [14] it was shown how the Bell states, the generalized GHZ states, some cluster-like states, and other states with varying degrees of entanglement may be generated directly from a braiding operator. Instead of using the Yang-Baxter equation, there we adopt a different approach. A new type of braiding operators were obtained by a unitary Jones representation of the braid group [18], which is constructed using a new representation of the Temperley-Lieb algebra (TLA) [19]. This new class of braiding operators generalizes the Bell matrix to multi-qubit systems, thus unifying the Hadamard, Bell and GHZ matrices within the same framework.

In recent years there is also a growing interest in generalising the braiding operators to quantum systems with higher ( $D$-level) dimensional Hilbert space, so-called qudits, both theoretical [20 32] and experimental [33]. This is due partly to the potential in enhanced security in quantum cryptography offered by qudit states as compared to qubit states [20], and partly to the potentially richer mathematical structures inherent in these systems. The Yang-Baxter approach was employed to construct braid operator for the bipartite qudit systems in 21 24]. In 26] multi-qudit cluster state was considered in a non-braid group approach based on the quantum plane algebra [34, 35]. Recently, this latter algebra was also employed in [25] to obtain a representation of braid operator for the multi-qudit systems.

In this work we would like to show how the braid group representation based on the Temperley-Lieb algebra considered in [14] for the qubit systems could be extended to multipartite qudit systems.

\footnotetext{
* Permanent address
} 
We shall first briefly review in Sect. II the relation between quantum entanglement and Jones representation of the braid group based on Temperley-Lieb algebra. We then discuss the unitary Jones representation of braid operators for the qubit systems in Sect. III, and extend it to qudit systems in Sect. IV. Braid quantum gates that can entangle multi-qudit states, and the generalized GHZ states are discussed in Sect. V. Sect. VI discusses how a general qudit state may be generated from one of its component state by a braid operator under appropriate condition. Sect. VII concludes the paper.

\section{BRAID GROUP AND QUANTUM ENTANGLEMENT}

Let us first briefly review the relation between braid group and quantum entanglement [10, 14].

The $m$-stranded braid group $B_{m}$ is generated by a set of elements $\left\{\sigma_{1}, \sigma_{2}, \ldots, \sigma_{m-1}\right\}$ with defining relations:

$$
\begin{aligned}
\sigma_{i} \sigma_{j} & =\sigma_{j} \sigma_{i}, \quad|i-j|>1 \\
\sigma_{i} \sigma_{i+1} \sigma_{i} & =\sigma_{i+1} \sigma_{i} \sigma_{i+1}, \quad 1 \leq i<m .
\end{aligned}
$$

To apply the braid group in quantum computing, one needs its unitary representations, because quantum gates are represented by unitary operators. For an $m$-qubit system the $2^{m} \times 2^{m}$ unitary representation of $B_{m}$ commonly employed is

$$
\sigma_{i}=I \otimes \ldots \otimes I \otimes R \otimes I \otimes \ldots \otimes I \quad(i=1 \ldots m-1)
$$

where $I$ is the $2 \times 2$ unit matrix and $R$ is a $4 \times 4$ unitary matrix that acts on both the $i$-th and $(i+1)$-th qubits; that is, occupying the $(i, i+1)$ position. This choice of the $\sigma_{i}$ 's satisfy the first of the two braid group relations in (11) automatically. To fulfil the second relation, $R$ must satisfy

$$
(R \otimes I)(I \otimes R)(R \otimes I)=(I \otimes R)(R \otimes I)(I \otimes R) .
$$

This relation is usually called the (algebraic) Yang-Baxter equation.

One of the simplest solutions of (3) that produces entanglement of states is the Bell matrix

$$
R=\frac{1}{\sqrt{2}}\left(\begin{array}{cccc}
1 & 0 & 0 & -1 \\
0 & 1 & -1 & 0 \\
0 & 1 & 1 & 0 \\
1 & 0 & 0 & 1
\end{array}\right)
$$

When acting on the standard basis $\{|00\rangle,|01\rangle,|10\rangle,|11\rangle\}, R$ generates the four maximally entangled Bell states $(|00\rangle \pm$ $|11\rangle) / \sqrt{2}$ and $(|01\rangle \pm|10\rangle) / \sqrt{2}$. Here we adopt the convention $|0\rangle=(1,0)^{t}$ and $|1\rangle=(0,1)^{t}$, where $t$ denotes the transpose. In the presence of local unitary transformations, $R$ is a universal gate [10]. The Bell matrix can be viewed as the bipartite generalisation of the Hadamard matrix

$$
H=\frac{1}{\sqrt{2}}\left(\begin{array}{cc}
1 & 1 \\
1 & -1
\end{array}\right),
$$

which when acts on the single qubit $|0\rangle$ and $|1\rangle$ gives a linear superposition of them.

In constructing the well-known Jones polynomials for knots and links, Jones [18] provided a new representation of the braid group based on what is essentially the TLA. The TLA, denoted by $T L_{m}(d)$, is defined, for an integer $m$ and a complex number $d$, to be the algebra generated by the unit element $I$ and the elements $h_{1}, h_{2}, \ldots, h_{m-1}$ satisfying the relations

$$
\begin{aligned}
h_{i} h_{j} & =h_{j} h_{i}, \quad|i-j|>1 ; \\
h_{i} h_{i \pm 1} h_{i} & =h_{i}, \quad 1 \leq i<m, \\
h_{i}^{2} & =d h_{i} .
\end{aligned}
$$

The Jones representation of the braid group is then given by (see eg., 36])

$$
\sigma_{i}=A h_{i}+A^{-1} I, \quad \sigma_{i}^{-1}=A^{-1} h_{i}+A I,
$$

where $A$ is a complex number given by $d=-A^{2}-A^{-2}$.

Generally the Jones representation is not unitary. However, if $A=e^{i \theta}(\theta \in[0,2 \pi))$ and all the $h_{i}$ 's are Hermitian $\left(h_{i}^{\dagger}=h_{i}\right)$, then the Jones representation is unitary. For $A=e^{i \theta}, d=-2 \cos 2 \theta$ is real. This also implies that $d^{2} \leq 4$.

Based on the unitary Jones representation of the 3 -stranded braid group $B_{3}$, we have generalized the Hadamard and Bell matrices to higher dimensions (i.e., to $n$ qubits), so that they generate generalized GHZ states from separable states directly [14]. In view of the recent interest in the qudit systems, here we would like to extend our construction in 14$]$ to the case of multi-qudit systems. 


\section{UNITARY JONES REPRESENTATION OF $B_{3}:$ 2-LEVEL QUBITS}

The construction in [14] is based on the following simple observation.

For single qubit systems, a simple unitary Jones representation of $B_{3}$ can be given by the TLA elements $h_{i}=d E_{i}$ $(i=1,2)$, where

$$
E_{1}=\left(\begin{array}{ll}
1 & 0 \\
0 & 0
\end{array}\right), \quad E_{2}=\left(\begin{array}{cc}
a^{2} & e^{-i \phi} a b \\
e^{i \phi} a b & b^{2}
\end{array}\right), \quad a^{2}+b^{2}=1 .
$$

Here $\phi$ is a phase angle. The $E_{i}$ 's satisfy

$$
\begin{aligned}
E_{i}^{2} & =E_{i} \\
E_{1} E_{2} E_{1} & =a^{2} E_{1} \\
E_{2} E_{1} E_{2} & =a^{2} E_{2} .
\end{aligned}
$$

With $a^{2}=d^{-2}, h_{i}$ 's as constructed from $E_{i}$ 's satisfy the TLA. For unitary representation of the braid group, one must have $d^{2} \leq 4$, and so $a^{2}=d^{-2} \geq 1 / 4$.

Now as $d$ and $a$ are real, in order that $h_{i}$ 's be Hermitian, we must have $b^{2}=1-1 / d^{2} \geq 0$. This implies $d^{2} \geq 1$ as well as the condition $d^{2} \leq 4$ mentioned before. Hence $\theta(\bmod 2 \pi)$ is restricted to be in the range $|\theta| \leq \pi / 6$, $|\theta-\pi / 2| \leq \pi / 6^{1}$ or $|\theta-\pi| \leq \pi / 6$. We shall assume $\theta$ to be in these domains hereafter. The special case of this representation with $\phi=0$ was employed previously in exploring the relation between quantum computing and the Jones polynomials [36, 37]).

To generalise the above representation of TLA to higher dimensions, we consider the following elements

$$
e_{1}=\left(\begin{array}{ll}
1 & 0 \\
0 & 0
\end{array}\right), e_{2}=\left(\begin{array}{cc}
a^{2} & 0 \\
0 & b^{2}
\end{array}\right), e_{3}=\left(\begin{array}{cc}
0 & e^{-i \phi} a b \\
e^{i \phi} a b & 0
\end{array}\right), \quad a^{2}+b^{2}=1
$$

Here $\phi$ is a phase angle. Define

$$
\begin{aligned}
E_{1} & \equiv \otimes_{j=1}^{k-1} I \otimes e_{1} \otimes_{j=k+1}^{n} I, \\
E_{2} & \equiv \otimes_{j=1}^{k-1} I \otimes e_{2} \otimes_{j=k+1}^{n} I \\
& +\otimes_{j=1}^{k-1} \lambda_{j} \otimes e_{3} \otimes_{j=k+1}^{n} \lambda_{j},
\end{aligned}
$$

where $\otimes_{j=1}^{m} \lambda_{j}=\lambda_{1} \otimes \lambda_{2} \otimes \cdots \otimes \lambda_{m}$. Here $\lambda_{j}$ is any Hermitian operator satisfying $\lambda_{j}^{2}=I$ (this requirement is to make $E_{2}^{2}=E_{2}$, which will be shown in the next paragraph). For example, $\lambda_{j}$ can be $I$, any one of the Pauli matrices $\sigma_{m}(m=1,2,3)$, or the Hadamard matrix $H$. The integer $n$ is the number of $2 \times 2$ matrices in the tensor products, and $k$ indicates the position of $e_{1}, e_{2}$ and $e_{3}$. The $E_{i}$ 's are $2^{n} \times 2^{n}$ matrices, and they reduce to (8) in the case of $n=k=1$. This freedom of choice in the $\lambda_{j}$ 's in our approach allows us to construct braiding operators that could generate different degrees of qubit entanglement.

The crucial point of the representation (11) is that the operators $e_{1}, e_{2}$ and $e_{3}$ satisfy the following identities:

$$
\begin{aligned}
e_{1}^{2} & =e_{1}, \\
e_{2}^{2}+e_{3}^{2} & =e_{2}, \\
e_{2} e_{3}+e_{3} e_{2} & =e_{3}, \\
e_{1} e_{2} e_{1} & =a^{2} e_{1}, \\
e_{1} e_{3} e_{1} & =0 \\
e_{2} e_{1} e_{2} & +e_{3} e_{1} e_{3}=a^{2} e_{2}, \\
e_{2} e_{1} e_{3} & +e_{3} e_{1} e_{2}=a^{2} e_{3} .
\end{aligned}
$$

Using these identities, one can easily check that $E_{i}$ 's satisfy (9). For instance, we can check if $E_{2}^{2}=E_{2}$ is satisfied. From Eq.(11) we have

$$
\begin{aligned}
E_{2}^{2} & =\otimes_{j=1}^{k-1} I \otimes e_{2}^{2} \otimes_{j=k+1}^{n} I+\otimes_{j=1}^{k-1} \lambda_{j}^{2} \otimes e_{3}^{2} \otimes_{j=k+1}^{n} \lambda_{j}^{2} \\
& +\otimes_{j=1}^{k-1} \lambda_{j} \otimes\left(e_{2} e_{3}+e_{3} e_{2}\right) \otimes_{j=k+1}^{n} \lambda_{j} .
\end{aligned}
$$

\footnotetext{
${ }^{1}$ We take this opportunity to add this range which was somehow neglected in 14 .
} 
With $e_{2}^{2}+e_{3}^{2}=e_{2}, e_{2} e_{3}+e_{3} e_{2}=e_{3}$, and $\lambda_{j}^{2}=I$, the equality $E_{2}^{2}=E_{2}$ is satisfied.

Hence, the operators $h_{i}=d E_{i}$ form a $2^{n} \times 2^{n}$ matrix realization of $T L_{3}(d)$. A unitary braid group representation is then obtained from the $h_{i}$ 's by the Jones representation. This new unitary braid representation generalizes the $2 \times 2$ matrices of (8) to $2^{n} \times 2^{n}$ matrices of (11) within the TLA $T L_{3}(d)$.

In the next section, we shall generalize the above construction to multipartite qudit systems.

\section{UNITARY JONES REPRESENTATION OF $B_{3}: D$-LEVEL QUDITS}

A $D$-level qudit state is denoted by $|s\rangle$, where $s=0,1, \ldots, D-1$, according to the convention that $|j\rangle=$ $(0,0, \ldots, 1,0, \ldots)^{t}$ has an entry " 1 " in the $(j+1)$-th row, and " 0 " elsewhere. The orthonormal computational basis of an $n$-qudit system is $\left\{\left|s_{1} s_{2} \cdots s_{n}\right\rangle\right\}$.

To extend the previous construction to the multipartite $D$-level qudits, we first note that $e_{1}, e_{2}$ and $e_{3}$ can be expressed as

$$
e_{1}=|0\rangle\left\langle 0\left|, e_{2}=a^{2}\right| 0\right\rangle\left\langle 0\left|+b^{2}\right| 1\right\rangle\langle 1|, \quad e_{3}=a b\left(e^{-i \varphi}|0\rangle\left\langle 1\left|+e^{i \varphi}\right| 1\right\rangle\langle 0|\right) .
$$

This inspires us to consider replacing the qubit $|1\rangle$ by the qudit $|l\rangle,(l=1,2, \ldots, D-1)$ in the definition of $e_{2}$ and $e_{3}$. Doing this we get $D-1$ sets of $e_{i}$ 's:

$$
\begin{aligned}
e_{1} & =|0\rangle\left\langle 0\left|, \quad e_{2}^{(l)}=a_{1}^{2}\right| 0\right\rangle\left\langle 0\left|+b_{1}^{2}\right| l\right\rangle\langle l|, \\
e_{3}^{(l)} & =a_{l} b_{l}\left(e^{-i \varphi_{l}}|0\rangle\left\langle l\left|+e^{i \varphi_{l}}\right| l\right\rangle\langle 0|\right), \quad l=1,2, \ldots, D-1 .
\end{aligned}
$$

Here $a_{l}, b_{l}(l=1,2)$ are real constants satisfying $a_{l}^{2}+b_{l}^{2}=1$, and $\varphi_{l}$ are arbitrary phases. The operator $e_{1}$ being the same for all the different sets.

It is easy to demonstrate that the identities in Eq. (12) are satisfied by the above sets of operators separately for each $l$.

With these sets of operators we can construct, for each value of $l$, the two corresponding TLA's generators as follows:

$$
\begin{aligned}
E_{1}^{(l)} & \equiv \otimes_{j=1}^{k-1} I \otimes e_{1} \otimes_{j=k+1}^{n} I, \\
E_{2}^{(l)} & \equiv \otimes_{j=1}^{k-1} I \otimes e_{2}^{(l)} \otimes_{j=k+1}^{n} I \\
& +\otimes_{j=1}^{k-1} \lambda_{j} \otimes e_{3}^{(l)} \otimes_{j=k+1}^{n} \lambda_{j}^{(l)} .
\end{aligned}
$$

Here $\lambda_{j}^{(l)}$ is any Hermitian operator satisfying $\lambda_{j}^{(l) 2}=I$. We can choose either $\lambda_{j}^{(l)}=I$, or

$$
\lambda_{j}^{(l)} \equiv\left|s_{j}\right\rangle\left\langle\tilde{s}_{j}^{(l)}|+| \tilde{s}_{j}^{(l)}\right\rangle\left\langle s_{j}\left|+\sum_{s_{j}^{\prime} \neq s_{j}, \tilde{s}_{j}^{(l)}}\right| s_{j}^{\prime}\right\rangle\left\langle s_{j}^{\prime}\right|,
$$

with the action $\lambda_{j}^{(l)}\left|s_{j}\right\rangle=\left|\tilde{s}_{j}^{(l)}\right\rangle, \lambda_{j}^{(l)}\left|\tilde{s}_{j}^{(l)}\right\rangle=\left|s_{j}\right\rangle$, and other qudits $\left|s_{j}^{\prime}\right\rangle\left(s_{j}^{\prime} \neq s_{j}, \tilde{s}_{j}^{(l)}\right)$ unchanged.

These operators satisfy the $T L_{3}(d)$ algebra with the constant $d$ given by $d_{l}^{2}=a_{l}^{-2}$ for each $l$. The corresponding braid operators are then given by the Jones representation

$$
\sigma_{i}^{(l)}=A_{l} d_{l} E_{i}^{(l)}+A_{l}^{-1} I, \quad \sigma_{i}^{(l)-1}=A_{l}^{-1} d_{l} E_{i}^{(l)}+A_{l} I,
$$

where $A_{l}$ are complex numbers determined by $d_{l}=-A_{l}^{2}-A_{l}^{-2}$. As before, $A_{l}$ being a pure phase requires $d_{l}^{2} \leq 4$, and $b_{l}^{2}=1-a_{l}^{2}=1-1 / d_{l}^{2} \geq 0$ implies $d_{l}^{2} \geq 1$, i.e., $1 \leq d_{l}^{2} \leq 4$, or $1 / 4 \leq a_{l}^{2} \leq 1$.

For each $l$, these $\sigma_{i}^{(l)}(i=1,2)$ furnish a $D^{n} \times D^{n}$ unitary representation of the braid group.

\section{BRAID QUANTUM GATES}

We define the unitary braiding transformation representing the action of the braid $\sigma_{1} \sigma_{2}$. This braiding operator is evaluated to be

$$
B_{0 l}(n, k) \equiv \sigma_{1}^{(l)} \sigma_{2}^{(l)}
$$




$$
\begin{aligned}
& =\otimes_{j=1}^{k-1} I \otimes\left(d_{l} a_{l}^{2}|0\rangle\left\langle 0\left|+\left(d_{l} b_{l}^{2}+A_{l}^{-2}\right)\right| l\right\rangle\left\langle l\left|+\sum_{m \neq 0, l} A_{l}^{-2}\right| m\right\rangle\langle m|\right) \otimes_{j=k+1}^{n} I \\
& +d_{l} a_{l} b_{l} \otimes_{j=1}^{k-1} \lambda_{j}^{(l)} \otimes\left(e^{i \varphi_{l}}|l\rangle\left\langle 0\left|-e^{-i \varphi_{l}} A_{l}^{4}\right| 0\right\rangle\langle l|\right) \otimes_{j=k+1}^{n} \lambda_{j}^{(l)} .
\end{aligned}
$$

Here we have explicitly included the pair $(n, k)$ to indicate that this operator acts on an $n$-qudit system, with the $k^{\text {th }}$ qudit being the "pivotal" qudit - linear superposition of states is effected at this position, while qudits at other positions are only modified by the $\lambda_{j}^{(l)}$ 's.

The action of $B_{0 l}(n, k)$ on the separable $n$-qudit state $\left|s_{1} s_{2} \cdots s_{n}\right\rangle\left(s_{j}=0,1, \ldots, D-1\right)$ is

$$
\begin{aligned}
B_{0 l}(n, k)\left|s_{1} s_{2} \cdots s_{k-1}, 0, s_{k+1} \cdots s_{n}\right\rangle= & \left(d_{l} a_{l}^{2}\right)\left|s_{1} s_{2} \cdots s_{k-1}, 0, s_{k+1} \cdots s_{n}\right\rangle \\
& +\left(e^{i \varphi_{l}} d_{l} a_{l} b_{l}\right)\left|\tilde{s}_{1}^{(l)} \tilde{s}_{2}^{(l)} \cdots \tilde{s}_{k-1}^{(l)}, l, \tilde{s}_{k+1}^{(l)} \cdots \tilde{s}_{n}^{(l)}\right\rangle \\
B_{0 l}(n, k)\left|s_{1} s_{2} \cdots s_{k-1}, l, s_{k+1} \cdots s_{n}\right\rangle= & \left(d_{l} b_{l}^{2}+A_{l}^{-2}\right)\left|s_{1} s_{2} \cdots s_{k-1}, l, s_{k+1} \cdots s_{n}\right\rangle \\
& +\left(e^{-i \varphi_{l}} A_{l}^{4} d_{l} a_{l} b_{l}\right)\left|\tilde{s}_{1}^{(l)} \tilde{s}_{2}^{(l)} \cdots \tilde{s}_{k-1}^{(l)}, 0, \tilde{s}_{k+1}^{(l)} \cdots \tilde{s}_{n}^{(l)}\right\rangle \\
B_{0 l}(n, k)\left|s_{1} s_{2} \cdots s_{k-1}, m, s_{k+1} \cdots s_{n}\right\rangle= & A_{l}^{-2}\left|s_{1} s_{2} \cdots s_{k-1}, m, s_{k+1} \cdots s_{n}\right\rangle, \quad m \neq 0, l .
\end{aligned}
$$

Here $\left|\tilde{s}_{j}^{(l)}\right\rangle \equiv \lambda_{j}^{(l)}\left|s_{j}\right\rangle(j=1, \ldots, k-1, k+1, \ldots, n)$. Thus under the action of $B_{0 l}(n, k)$, the separable $n$-qudit state $\left|s_{1} s_{2} \cdots 0 \cdots s_{n}\right\rangle$ is superimposed on the state $\left|\tilde{s}_{1} \tilde{s}_{2} \cdots l \cdots \tilde{s}_{n}\right\rangle$, and likewise $\left|s_{1} s_{2} \cdots, l, \cdots s_{n}\right\rangle$ is superimposed on the state $\left|\tilde{s}_{0} \tilde{s}_{2} \cdots, 0, \cdots \tilde{s}_{n}\right\rangle$. We have used the subscript $\{0 l\}$ in $B_{0 l}(n, k)$ to indicate this fact. The state in (20) is normalized, as $\left(d_{l} a_{l}^{2}\right)^{2}+\left|e^{i \phi_{l}} d_{l} a_{l} b_{l}\right|^{2}=1$. Depending on the choice of the set of $\lambda_{j}^{(l)}$ 's, the resulting state will have varying degree of entanglement. In particular, if all $\lambda_{j}^{(l)}=I$, then the resulting state is separable, and $B_{0 l}(n, k)$ is simply a local unitary transformation.

\section{A. Successive superpositions of states}

We now consider successive actions of braid operators $B_{0 l}(n, k)(l=1,2, \ldots, D-1)$ on the separable $n$-qudit $\left|\phi_{0}\right\rangle \equiv\left|s_{1} s_{2} \cdots s_{k-1}, 0, s_{k+1} \cdots s_{n}\right\rangle$.

Clearly we have from Eq. (20)

$$
\left|\phi_{1}\right\rangle \equiv B_{01}(n, k)\left|\phi_{0}\right\rangle=\left(d_{1} a_{1}^{2}\right)\left|s_{1} s_{2} \cdots s_{k-1} 0 s_{k+1} \cdots s_{n}\right\rangle+\left(e^{i \varphi_{1}} d_{1} a_{1} b_{1}\right)\left|\tilde{s}_{1}^{(1)} \tilde{s}_{2}^{(1)} \cdots \tilde{s}_{k-1}^{(1)} 1 \tilde{s}_{k+1}^{(1)} \cdots \tilde{s}_{n}^{(1)}\right\rangle .
$$

Acting $B_{02}(n, k)$ on the state $\left|\phi_{1}\right\rangle$ gives

$$
\begin{aligned}
\left|\phi_{2}\right\rangle \equiv B_{02}(n, k) B_{01}(n, k)\left|\phi_{0}\right\rangle= & \left(d_{2} a_{2}^{2}\right)\left(d_{1} a_{1}^{2}\right)\left|s_{1} s_{2} \cdots s_{k-1} 0 s_{k+1} \cdots s_{n}\right\rangle \\
& +A_{2}^{-2}\left(e^{i \varphi_{1}} d_{1} a_{1} b_{1}\right)\left|\tilde{s}_{1}^{(1)} \tilde{s}_{2}^{(1)} \cdots \tilde{s}_{k-1}^{(1)} 1 \tilde{s}_{k+1}^{(1)} \cdots \tilde{s}_{n}^{(1)}\right\rangle \\
+ & \left(e^{i \varphi_{2}} d_{2} a_{2} b_{2}\right)\left(d_{1} a_{1}^{2}\right)\left|\tilde{s}_{1}^{(2)} \tilde{s}_{2}^{(2)} \cdots \tilde{s}_{k-1}^{(2)} 2 \tilde{s}_{k+1}^{(2)} \cdots \tilde{s}_{n}^{(2)}\right\rangle .
\end{aligned}
$$

It is clear that the successive actions of $B_{0 l}(n, k)$ 's are to linearly superimpose the states $\left|s_{1} s_{2} \ldots s_{k-1} 0 s_{k+1} \cdots s_{n}\right\rangle,\left|\tilde{s}_{1}^{(1)} \tilde{s}_{2}^{(1)} \cdots \tilde{s}_{k-1}^{(1)} 1 \tilde{s}_{k+1}^{(1)} \cdots \tilde{s}_{n}^{(1)}\right\rangle, \ldots,\left|\tilde{s}_{1}^{(1)} \tilde{s}_{2}^{(l)} \ldots \tilde{s}_{k-1}^{(l)} l \tilde{s}_{k+1}^{(l)} \ldots \tilde{s}_{n}^{(l)}\right\rangle \quad(l=1,2, \ldots, D-1)$ successively. Hence

$$
\begin{aligned}
\left|\phi_{l}\right\rangle \equiv & B_{0 l}(n, k) B_{0, l-1}(n, k) \cdots B_{02}(n, k) B_{01}(n, k)\left|\phi_{0}\right\rangle \\
= & \alpha_{l 0}\left|s_{1} s_{2} \cdots s_{k-1} 0 s_{k+1} \cdots s_{n}\right\rangle+\sum_{p=1}^{l} \alpha_{l p}\left|\tilde{s}_{1}^{(p)} \tilde{s}_{2}^{(p)} \cdots \tilde{s}_{k-1}^{(p)} p \tilde{s}_{k+1}^{(p)} \cdots \tilde{s}_{n}^{(j)}\right\rangle \\
& \quad \alpha_{l r}: \text { complex constants; } \quad l=1,2, \ldots, D-1 ; \quad r=0,1, \ldots, l .
\end{aligned}
$$

Depending on the choice of the set of operators $\lambda_{j}^{(l)}$ 's, the states $\left|\phi_{l}\right\rangle$ could be separable (e.g., if all $\lambda_{j}^{(l)}=I$ ), partially entangled, or completely entangled. Also, as $B_{0 l}(n, k)$ is unitary, normalizability is maintained at each transformation. This can be checked explicitly. Acting $B_{0, l+1}(n, k)$ on $\left|\phi_{l}\right\rangle$, we get

$$
\begin{aligned}
\left|\phi_{l+1}\right\rangle & \equiv B_{0, l+1}(n, k)\left|\phi_{l}\right\rangle \\
& =\left(d_{l+1} a_{l+1}^{2} \alpha_{l 0}\right)\left|s_{1} s_{2} \cdots s_{k-1}, 0, s_{k+1} \cdots s_{n}\right\rangle
\end{aligned}
$$




$$
\begin{aligned}
& +\sum_{p=1}^{l}\left(A_{l+1}^{-2} \alpha_{l p}\right)\left|\tilde{s}_{1}^{(p)} \tilde{s}_{2}^{(p)} \cdots \tilde{s}_{k-1}^{(p)}, p, \tilde{s}_{k+1}^{(p)} \cdots \tilde{s}_{n}^{(p)}\right\rangle \\
& +\left(e^{i \varphi_{l+1}} d_{l+1} a_{l+1} b_{l+1} \alpha_{l 0}\right)\left|\tilde{s}_{1}^{(l+1)} \tilde{s}_{2}^{(l+1)} \cdots \tilde{s}_{k-1}^{(l+1)}, l+1, \tilde{s}_{k+1}^{(l+1)} \cdots \tilde{s}_{n}^{(l+1)}\right\rangle .
\end{aligned}
$$

Using the relations $a_{l+1}^{2}+b_{l+1}^{2}=1$ and $d_{l+1}^{2} a_{l=1}^{2}=1$, one can check that

$$
\left\langle\phi_{l+1} \mid \phi_{l+1}\right\rangle=\sum_{s=0}^{l}\left|\alpha_{l s}\right|^{2}=\left\langle\phi_{l} \mid \phi_{l}\right\rangle
$$

So $\left|\phi_{l+1}\right\rangle$ is normalized if $\left|\phi_{l}\right\rangle$ is. It is obvious that for $l=1$, the state $\left|\phi_{1}\right\rangle$ is normalized. Hence by induction the states $\left|\phi_{l}\right\rangle$ generated by the braid quantum gates $B_{0 l}(n, k)$ 's are normalized.

\section{B. Coefficients of $\left|\phi_{l}\right\rangle$}

Eq. (24) relates the coefficients of $\left|\phi_{l+1}\right\rangle$ to those of $\left|\phi_{l}\right\rangle$. In this and the next subsection, for simplicity of notation, we shall take $d_{l}=1 / a_{l}$ (i.e., we take the positive sign of the relation $d_{l}^{2}=a_{l}^{-2}$ ), and also set all the phase angles to zero, $\varphi_{l}=0$. Then Eq. (24) gives

$$
\begin{aligned}
\alpha_{l+1,0} & =a_{l+1} \alpha_{l 0} ; \\
\alpha_{l+1, p} & =A_{l+1}^{-2} \alpha_{l p}, \quad p=1,2, \ldots, l ; \\
\alpha_{l+1, l+1} & =b_{l+1} \alpha_{l 0} .
\end{aligned}
$$

For the initial separable state $\left|s_{1} s_{2} \cdots s_{k}, 0, s_{k=1} \cdots s_{n}\right\rangle$, the initial value of the coefficient is $\alpha_{00}=1$. Then from Eq. (26) we have the solution of the coefficients $\alpha_{0 s}(s=0,1, \ldots, l)$ :

$$
\begin{aligned}
\alpha_{l 0}= & a_{l} a_{l-1} \cdots a_{2} a_{1}, \\
\alpha_{l 1}= & A_{l}^{-2} A_{l-1}^{-2} \cdots A_{3}^{-2} A_{2}^{-2} b_{1}, \\
\alpha_{l 2}= & A_{l}^{-2} A_{l-1}^{-2} \cdots A_{3}^{-2} b_{2} a_{1}, \\
\alpha_{l 3}= & A_{l}^{-2} A_{l-1}^{-2} \cdots A_{4}^{-2} b_{3} a_{2} a_{1}, \\
& \vdots \\
\alpha_{l l}= & b_{l} a_{l-1} \cdots a_{2} a_{1} .
\end{aligned}
$$

\section{Generalized GHZ qudit states}

We define the $l$-level generalized GHZ $n$-qudit state as

$$
|G H Z\rangle_{l} \equiv \sum_{r=0}^{l} \alpha_{l r}|r r r \cdots r r r\rangle, \quad l=1, \ldots, D-1,
$$

for which all the component states have the same probability to be measured, i.e., with $\left|\alpha_{l r}\right|^{2}=1 /(l+1)$ for all $r$. These are the maximally entangled multi-qudit states.

We show here that by appropriately choosing the initial state $\left|\phi_{0}\right\rangle$, the set of operators $\lambda_{j}^{(p)}$ 's, and the sets of scalars $\left\{a_{p}, b_{p}\right\}(p=1, \ldots, l)$, one can make $\left|\phi_{l}\right\rangle$ in Eq. (23) a GHZ state.

We start with the initial state $\left|\phi_{0}\right\rangle=|00 \cdots 00\rangle$, i.e., choose all $\left|s_{j}\right\rangle=|0\rangle,(j=1,2, \ldots, k-1, k+1, \ldots, n)$. Then select the $\lambda_{j}^{(p)}$ to be

$$
\lambda_{j}^{(p)}=|0\rangle\langle p|+| p\rangle\left\langle 0\left|+\sum_{q \neq 0, p}\right| q\right\rangle\langle q|, \quad p=1, \ldots, l .
$$

These $\lambda_{j}^{(p)}$ change the $j$-qudit $|0\rangle_{j}$ in $\left|\phi_{0}\right\rangle$ to $|p\rangle_{j}, \lambda_{j}^{(p)}|0\rangle_{j}=|p\rangle_{j}$. 
Next we have to solve the constraints $\left|\alpha_{l r}\right|^{2}=1 /(l+1)$ for the required sets of $\left\{a_{p}, b_{p}\right\}$. From Eq. (27) it is found that the solution is given by

$$
\begin{array}{rlrl}
a_{1}^{2}=\frac{l}{l+1}, & b_{1}^{2} & =\frac{1}{l+1}, \\
a_{2}^{2}=\frac{l-1}{l}, & b_{2}^{2}=\frac{1}{l} \\
\vdots & \\
a_{j}^{2}=\frac{l-j+1}{l-j+2}, & b_{j}^{2}=\frac{1}{l-j+2}, \\
\vdots & \\
a_{l}^{2}=\frac{1}{2}, & b_{l}^{2}=\frac{1}{2} .
\end{array}
$$

To fulfil the conditions $1 \leq d_{j}=1 / a_{j} \leq 2$, we must take the positive roots for $a_{j}$, i.e., $1 / \sqrt{2} \leq a_{j}=$ $\sqrt{(l-j+1) /(l-j+2)}<1$. The sign of $b_{j}$ can be chosen differently for different GHZ states. The phase $A_{l}^{-2}$ is solved from $d_{l}=-A_{l}^{2}-A_{l}^{-2}=1 / a_{l}$.

With these parameters, one has from Eq. (23)

$$
|G H Z\rangle_{l}=B_{0 l}(n, k) B_{0, l-1}(n, k) \cdots B_{02}(n, k) B_{01}(n, k)|00 \cdots 00\rangle .
$$

Hence the generalised $l$-level GHZ $n$-qudit state can be generated from the original state $|00 \cdots 00\rangle$.

\section{GENERATION OF A GENERAL STATE FROM ONE OF ITS COMPONENT STATE}

In this section we demonstrate that there exists a TLA-based unitary braid quantum gate which connects a general multi-qudit state and one of its component basis states, if the coefficient of the component state is such that the square of its norm is no less than $1 / 4$.

For clarity of presentation in this section, we shall replace the notation $\left|s_{1} s_{2} s_{3} \cdots\right\rangle$ for the orthonormal computational basis of $n$-qudits by $\left\{|i j k \cdots\rangle_{n}\right\}(i, j, k, \ldots=0,1, \ldots, D-1)$.

Now consider a general normalized state $|\psi\rangle=\sum_{i, j, k, \cdots=0}^{D-1} \alpha_{i j k \cdots}|i j k \cdots\rangle$. Let $|\psi\rangle$ contains a component state, $|a b c \cdots\rangle$, say, i.e., $|\psi\rangle=\cdots+\alpha_{a b c \cdots}|a b c \cdots\rangle+\cdots$. Next we construct the following two projection operators from $|a b c \cdots\rangle$ and $|\psi\rangle$,

$$
\begin{aligned}
& E_{1}=|a b c \cdots\rangle\langle a b c \cdots|, \\
& E_{2}=|\psi\rangle\langle\psi| .
\end{aligned}
$$

By construction $E_{1}$ and $E_{2}$ are Hermitian, and satisfy the first set of equations of TLA in Eq. (9). It is straightforward to show that the second and the third set of Eq. (9) are also satisfied,

$$
\begin{aligned}
& E_{1} E_{2} E_{1}=\left|\alpha_{a b c \cdots}\right|^{2} E_{1}, \\
& E_{2} E_{1} E_{2}=\left|\alpha_{a b c \cdots}\right|^{2} E_{2},
\end{aligned}
$$

obviously with $\left|\alpha_{a b c \cdots}\right|^{2}$ playing the role of the scalar $d^{-2}$ of the TLA, $d^{2}=\left|\alpha_{a b c \cdots}\right|^{-2}$. Now in order for the TLA constructed from $E_{1}$ and $E_{2}$ to be unitary, one must have $1 \leq d^{2} \leq 4$. This requires $1 / 4 \leq\left|\alpha_{a b c \cdots}\right|^{2} \leq 1$. The r.h.s. of the constraint is always satisfied as $\left|\alpha_{a b c \ldots}\right|^{2} \leq 1$ for a normalized $|\psi\rangle$.

We can then form the braid generators $\sigma_{1}$ and $\sigma_{2}$ by the Jone representation as discussed before. Applying the braid operator $\sigma_{1} \sigma_{2}$ on the separable state $|a b c \cdots\rangle$, we get

$$
\sigma_{1} \sigma_{2}|a b c \cdots\rangle=d \alpha_{a b c \cdots}^{*}|\psi\rangle+\left(d+A^{-2}+\left(d^{2}\left|\alpha_{a b c \cdots}\right|^{2}\right) A^{2}\right)|a b c \cdots\rangle .
$$

Since $d^{2}\left|\alpha_{a b c \cdots}\right|^{2}=1, d=-A^{2}-A^{-2}$ and $d \alpha_{a b c \ldots}^{*}=\exp \left(i \arg \alpha_{a b c \cdots}\right)$, we finally obtain,

$$
\sigma_{1} \sigma_{2}|a b c \cdots\rangle=\exp \left(i \arg \alpha_{a b c \cdots}\right)|\psi\rangle \text {. }
$$

So the braid quantum gate generates the state $|\psi\rangle$, up to a pure phase, from its component state $|a b c \cdots\rangle$. Of course, one can always factor out the phase as a global phase from the definition of $|\psi\rangle$. In this case, $\alpha_{a b c \cdots}$ is a real constant, and we will have $\sigma_{1} \sigma_{2}|a b c \cdots\rangle=|\psi\rangle$.

For the $l$-level generalized GHZ state defined in Eq. (28), the coefficients all give equal value, $\left|\alpha_{l r}\right|^{2}=1 /(l+1)$. For $\left|\alpha_{l r}\right|^{2}=1 /(l+1) \geq 1 / 4$, one must have $l+1=D \leq 4$. Thus GHZ state can be generated from its component state by the method presented here only for qudits with level $D \leq 4$. 


\section{SUMMARY AND DISCUSSION}

In this paper we have constructed braid operators that could generate entangled $n$-partite $D$-level qudit states, using a braid group representation based on the Temperley-Lieb algebra. $D$ different sets of $D^{n} \times D^{n}$ unitary representation $\left\{\sigma_{1}^{(l)}, \sigma_{2}^{(l)}\right\}(l=1,2, \ldots, D)$ of the braid generators were presented. Using these generators the desired braid quantum gates $B_{0 l}(n, k)$ were obtained, where the $k$-qudit acts as the pivotal qudit. Linear superposition of states was effected at this position, while the state of qudits at the other positions were transformed to another state by certain exchange operators. Multi-qudit states of varying degree of entanglement can be generated by various combinations of $B_{0 l}(n, k)$ 's with different $l$ and $k$. Particularly, we showed that the generalized GHZ states could be obtained directly without resorting to further local unitary transformations as in the Yang-Baxter approach.

For definiteness we have presented our construction of the braid generators and the braid quantum gates using $|0\rangle$ as the pivotal qudit. This is not necessary. In fact any qudit, say $|q\rangle,(q=1,2, \ldots, D-1)$, can serve as the pivotal qudit. To obtain the corresponding braid generators and the braid quantum gates, we need only to exchange $|0\rangle$ and $|q\rangle$ in the expressions presented in this work. For instance, the braid quantum gate $B_{0 l}(n, k)$ in Eq. (19) becomes

$$
\begin{aligned}
B_{q l}(n, k) & =\otimes_{j=1}^{k-1} I \otimes\left(d_{l} a_{l}^{2}|q\rangle\left\langle q\left|+\left(d_{l} b_{l}^{2}+A_{l}^{-2}\right)\right| l\right\rangle\left\langle l\left|+\sum_{m \neq q, l} A_{l}^{-2}\right| m\right\rangle\langle m|\right) \otimes_{j=k+1}^{n} I \\
& +d_{l} a_{l} b_{l} \otimes_{j=1}^{k-1} \lambda_{j}^{(l)} \otimes\left(e^{i \varphi_{l}}|l\rangle\left\langle q\left|-e^{-i \varphi_{l}} A_{l}^{4}\right| q\right\rangle\langle l|\right) \otimes_{j=k+1}^{n} \lambda_{j}^{(l)}, \quad l \neq q
\end{aligned}
$$

One then applies $B_{q l}(n, k)$ on the separable state $\left|s_{1} s_{2} \cdots, q, \cdots s_{n}\right\rangle$ to generate other linear superimposed states.

We have also pointed out an interesting observation that for a general multi-qudit state there exists a unitary braid quantum gate based on the Temperley-Lieb algebra that connects the multi-qudit with one of its component basis state, provided the coefficient of the component state is such that the square of its norm is no less than $1 / 4$.

\section{Acknowledgments}

The present research is partially supported by the Ministry of Science and Technology (MoST) of the Republic of China under Grants 102-2112-M-032-003-MY3 and 105-2918-I-032-001 (CLH), and the Grant-in-Aid for Scientific Research No. $15 \mathrm{~K} 05204$ (TD). This work was done during CLH's visit at the Ochanomizu University. He would like to thank the members of the Department of Physics, particularly E. Uehara, E. Nozawa, C. Matsuyama, and N. Oshima, for the hospitality extended to him.

[1] A. Yu Kitaev, Ann. Phys. 303 (2003) 2.

[2] A. Yu Kitaev, Ann. Phys. 321 (2006) 2.

[3] M. Freedman, M. Larsen, and Z. Wang, Comm. Math. Phys. 227 (2002) 605.

[4] C. Nayak, S. Simon, A. Stern, M. Freedman, and S. Das Sarma, Rev. Mod. Phys. 80 (2008) 1083.

[5] A. Yu Kitaev, Phys. Usp. 44 (2001) 131.

[6] D.A. Ivanov, Phys. Rev. Lett. 86 (2001) 268.

[7] F. Wilczek, Nature Phys. 5 (2009) 614.

[8] L. Fu and C. L. Kane. Phys. Rev. Lett. 100 (2008) 096407.

[9] C. W. J. Beenakker, Ann. Rev. Con. Mat. Phys. 4 (2013) 113.

[10] L.H. Kauffman and S.J. Lomonaco Jr., New J. Phys. 6 (2004) 134.

[11] Y. Zhang, L.H. Kauffman, and M.-L. Ge, Int. J. Quant. Info. 3 (2005) 669.

[12] J.-L. Chen, K. Xue, and M.-L. Ge, Phys. Rev. A 76 (2007) 042324.

[13] Y. Zhang, N. Jing, and M.-L. Ge, J. Phys. A41 (2008) 055310.

[14] C.-L. Ho, A.I. Solomon, and C.-H. Oh, Europhys. Lett.92 (2010) 30002.

[15] G. Wang, K. Xue, C. Sun, B. Liu, Y. Liu, and Y. Zhang, Phys. Lett. A 379 (2015) 1.

[16] D.M. Greenberger, M.A. Horne, and A. Zeilinger, "Going Beyond Bell's Theorem", in Bell's Theorem, Quantum Theory, and Conceptions of the Universe, M. Kafatos (Ed.), pg. 69-72 (Kluwer, Dordrecht, 1989).

[17] H. J. Briegel and R. Raussendorf , Phys. Rev. Lett. 86 (2001) 910.

[18] V.F.R. Jones, Bull. Am. Math. Soc. 12 (1985) 103.

[19] H. Temperley and E. Lieb, Proc. Roy. Soc. (Landon) A 322 (1971) 251.

[20] N.J. Cerf, M. Bourennane, A. Karlsson, and N. Gisin, Phys. Rev. Lett. 88 (2002) 127902.

[21] B. Abdesselam, A. Chakrabarti, V.K. Dobrev, and S.G. Mihov, J. Math. Phys. 48 (2007) 053508. 
[22] G. Wang, K. Xue, C. Sun, C. Zhou, T. Hu, and Q. Wang, arXiv:0903.3711

[23] C. Sun, G. Wang, T. Hu, C. Zhou, Q. Wang, and K. Xue, Int. J. Quant. Info. 7 (2009) 1285.

[24] C. Sun, K. Xue, G. Wang, and C. Wu, arXiv:1001.4587.

[25] G. Wang, C. Sun, C. Wu, B. Liu, Y. Zhang, and K. Xue, Europhys. Lett. 108 (2014) 10001.

[26] D. Zhou, B. Zeng, Z. Xu, and C. Sun, Phys. Rev. A 68 (2003) 062303.

[27] J. Ryu, C. Lee, M. Zukowski, and J. Lee, Phys. Rev. A 88 (2013) 042101.

[28] G. Gualdi, D. Licht, D.M. Reich, C.P. Koch, Phys. Rev. A 90 (2014) 032317.

[29] Z. Gedik, I. A. Silva, B. akmak, G. Karpat, E. L. G. Vidoto, D.O. Soares-Pinto, E.R. deAzevedo, F.F. Fanchini, Scientific Reports 5 (2015) 14671.

[30] S.K. Goyal, T. Konrad, Scientific Reports 3 (2013) 3548.

[31] H. F. Chau, C. Wong, Q. Wang, T. Huang, Qudit-Based Measurement-Device-Independent Quantum Key Distribution Using Linear Optics. arXiv:1608.08329 [quant-ph].

[32] P. Ługiewicz, A. Frydryszak, L. Jakóbczyk, J. Phys. A 50 (2017) 245301.

[33] B. Marques, A. A. Matoso, W. M. Pimenta, A. J. Gutirrez-Esparza, M. F. Santos, S. Pádua, Scientific Reports 5 (2015) 16049.

[34] H. Weyl, The theory of Groups and Quantum Mechanics, Dover, New York, 1950.

[35] J. Schwinger, Proc. Natl. Acad. Sci. U.S.A. 46 (1960) 570.

[36] L.H. Kauffman, AMS Contemp. Math. Series 305 (2002) 101.

[37] L.H. Kauffman and S.J. Lomonaco Jr., J. Knot Theory Ramifications 16 (2007) 267. 